\title{
Collision between a Pedestrian and Tram - Pilot Experiment
}

\author{
Lopot, F. ${ }^{1}$, Kubový, P. ${ }^{1}$, Jelen, K. ${ }^{1}$, Šorfová, M. ${ }^{1}$, Tlapáková, E. ${ }^{1}$, Rulc, V. ${ }^{2}$, Purš, H. ${ }^{2}$, Ježdík, R. ${ }^{3}$, Svoboda, M. ${ }^{4}$ \\ ${ }^{1}$ Faculty of Physical Education and Sports, Charles University, José Martího 31, 162 52, Prague 6, E-mails: \\ lopot@ftvs.cuni.cz,kubovy@ftvs.cuni.cz,jelen@ftvs.cuni.cz, sorfova@ftvs.cuni.cz, tlapakova@ftvs.cuni.cz \\ ${ }^{2}$ Advanced Engineering, s. r. o. Na Ostrohu 2405/16, 160 00, Prague 6, E-mails: vrulc@advanced-eng.cz, \\ hpurs@advanced-eng.cz \\ ${ }^{3}$ VÚKV a.s., Bucharova 1314/8 Stodůlky, 150 00, Prague 5, E-mail: jezdik@vukv.cz \\ ${ }^{4}$ Faculty of Mechanical Engineering, Jan Evangelista Purkyně University in Ústí nad Labem, Pasteurova 1, 400 01, Ústí \\ nad Labem, E-mail: martin.svoboda@ujep.cz
}

This article intends to present the first results of a long-term research project, which will result in developing a validated model of a pedestrian for the simulation of crash tests involving tram fronts and, where applicable, the fronts of other urban rail vehicles. The current phase of research includes results of the pilot experiment with a crash-test dummy, and these results supplement the results from simulations, thus demonstrating how important it is to pay special attention to the individual stages of a collision event and how important the localisation of and moulding by an individual tram's front panels are for the nature of the monitored stages. In the first stage, inertia of individual body segments plays a significant role, with the primary contact taking place between the tram's bumper and dummy's thigh. The dummy subsequently "takes the shape" of the tram's front with progressive bumps to shoulders and head following. At that moment, the tram brakes, and the dummy begins to disentangle from the front panel. The friction force between the dummy's soles and the surface of the rail track is very significant for the nature of this second stage of the collision event .The dummy then hits the ground. It is an accelerated fall, and under the given conditions, it is the stage that has the most devastating impact on the dummy. The simulation made shows the way to modify this dangerous stage to be less harmful to a pedestrian involved in a tram collision.

Keywords: tram, pedestrian, crash test, simulation, passive safety, FEM /finite element method/

\section{Introduction}

Current urban transport planning includes greater pressure to develop technical solutions to mitigate the negative impacts of public transport vehicle collisions on pedestrians. These collisions have two observable causes: the increased volume of public transport in urban agglomerations due to efforts to reduce local emissions, and pedestrians' evident inattentiveness from the use of mobile technologies. A particularly intensive development over the past few years can be seen with urban rail vehicles, which are particularly dangerous for pedestrians as vehicles cannot avoid a collision through evasive action. Upon the initiative of European institutes and manufacturers developing rail vehicles not only for urban transport, a methodology for testing the passive safety of vehicle bodies is being developed where the tests are based on frontal collisions between the vehicle and a pedestrian. Extensive accident databases across EU countries [1] were used as supporting material for setting the test conditions. Outputs of the tests should then be used, among other things, as a basis for new structures of rail vehicle fronts. For this reason, one of the essential steps is to prepare a validated model of a pedestrian for virtual experimentation, which is an integral part of the construction process today. This study provides findings about a crash test performed with an older type of tram (selected for its front shape) as well as the results of a simulation for the same type with the front's modified lower part. The objective is to illustrate how important it is to monitor the stages of a collision event in order to evaluate its actual dangers to a pedestrian hit by a given vehicle.

\section{Experiment}

For the first of the planned series of crash tests, we chose a T6A5 ČKD Tatra tram with a "classic" front from the 1980s. The tests were conducted at speeds of 10 $\mathrm{km} /$ hour and $20 \mathrm{~km} /$ hour. The Hybrid III dummy representing a typical male (Jasti Hybrid III, 50th Percentile Male with Pedestrian kit) was placed upright and side on in the way of a tram within a zone corresponding to one third of the tram's width. This arrangement complies with the current version of said European regulation which is currently under preparation [1]. For data recording, a high-speed camera with a recording frequency of 12,000 $\mathrm{Hz}$ and the Qualisys 3D cinematic system in an eightcamera configuration with a recording frequency of 300 $\mathrm{Hz}$, were used. A second high-speed camera (500 fps) with a wide angle of the collision event was also used in the experiment; however, data from that camera were not included in this analysis. Accelerometry and other sensor technologies were not used with the dummy as it was a pilot measurement, and no estimate of the dummy's destruction was available. The dummy used is also not intended for studying direct side-on impacts; therefore, one of the measurement outputs is to verify the dummy's usability for this type of measurement. In spite of these limitations, the experiment bore very interesting results. See the measurement site layout in Figure 1.

The rail tracks are indicated by the black lines. The experiment was performed on a straight part of the testing 
rail track with a total length of about $200 \mathrm{~m}$. The collision was staged approximately in the middle of the track. The green lines indicate the areas tracked by Qualisys; the blue lines show the field of vision of a distant high-speed camera, and the red lines show the angle field of a second high-speed camera.

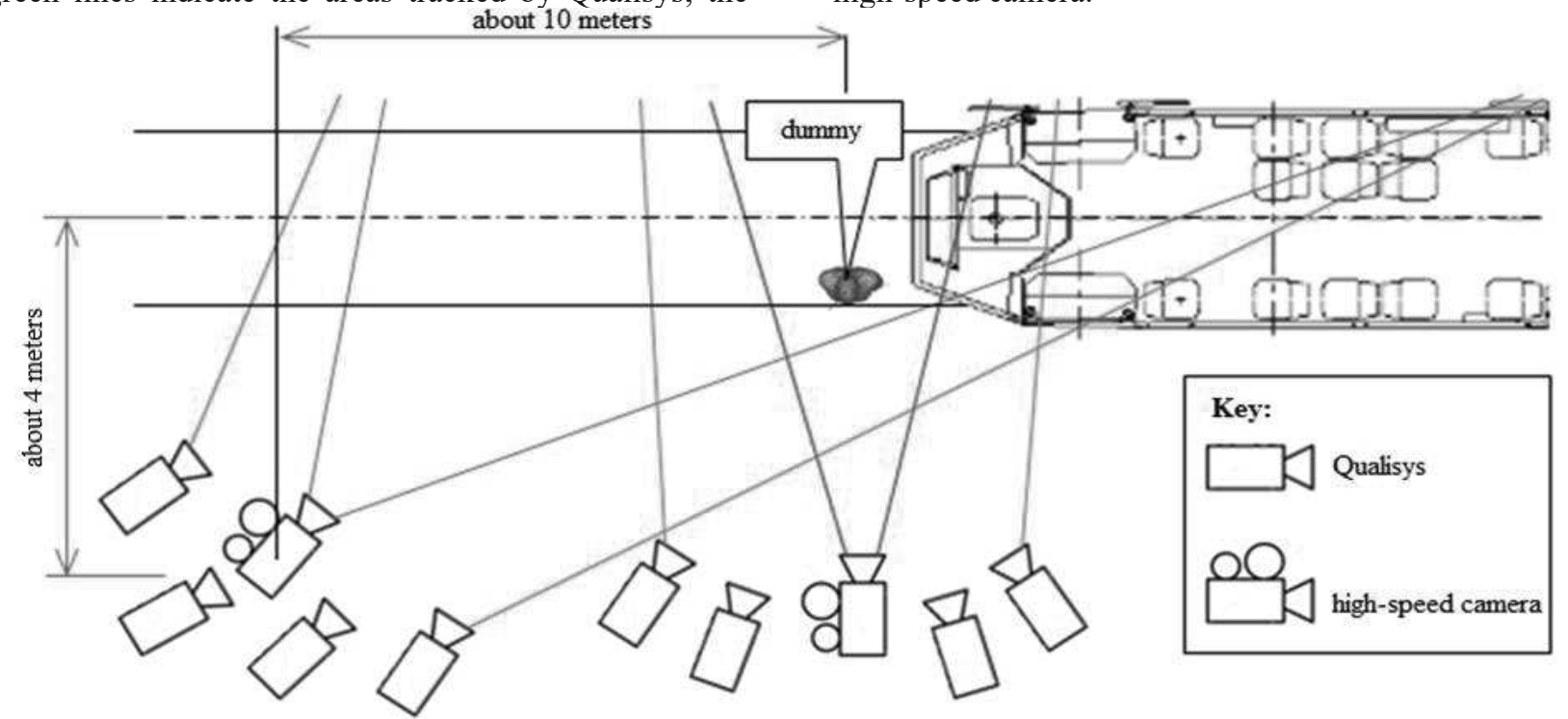

Fig. 1 Measurement site layout

As part of the preliminary preparation of virtual model validation, we simulated the impact on the tram's front processed in the Altair HyperWorks environment. For the simulation, we used a so-called multi-body dummy model, i.e. a dummy composed of individual segments not allowing for any detailed studying of the segments' deformation field. The dummy's surface, however, is a network of elements that accurately represent a geometrical shape of the dummy's body. The model of the tram's front was the end element (with 5,948 3D elements, 184,359 2D elements, and 320 1D elements), based on the known material characteristics of individual front panels and other structural components of the front.

The following series of images document the course of the test made at $20 \mathrm{~km} /$ hour. Please note that the abovedescribed staging of the collision event is easily identifiable for all measurements made. Times of shots were included in the image captions for the benefit of the reader. You can see point trajectories on the dummy monitored by Qualisys.

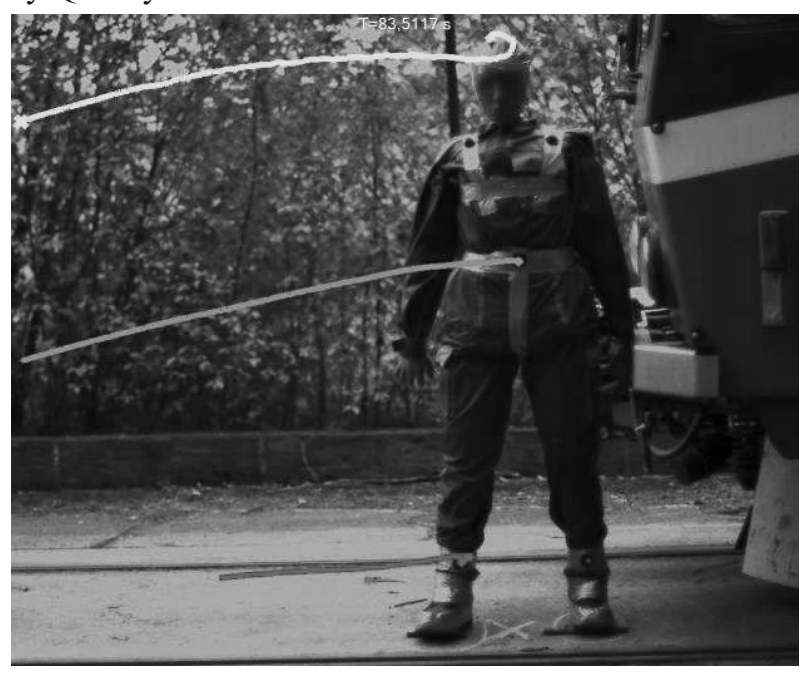

Fig. 2 Time of the first contact (time 0s)

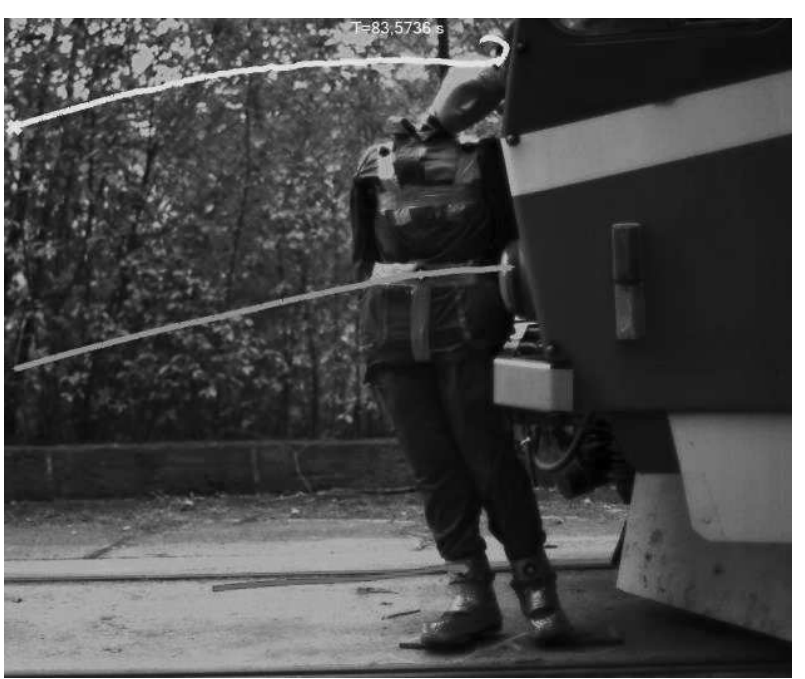

Fig. 3 First stage of collision action (time 0.03s)

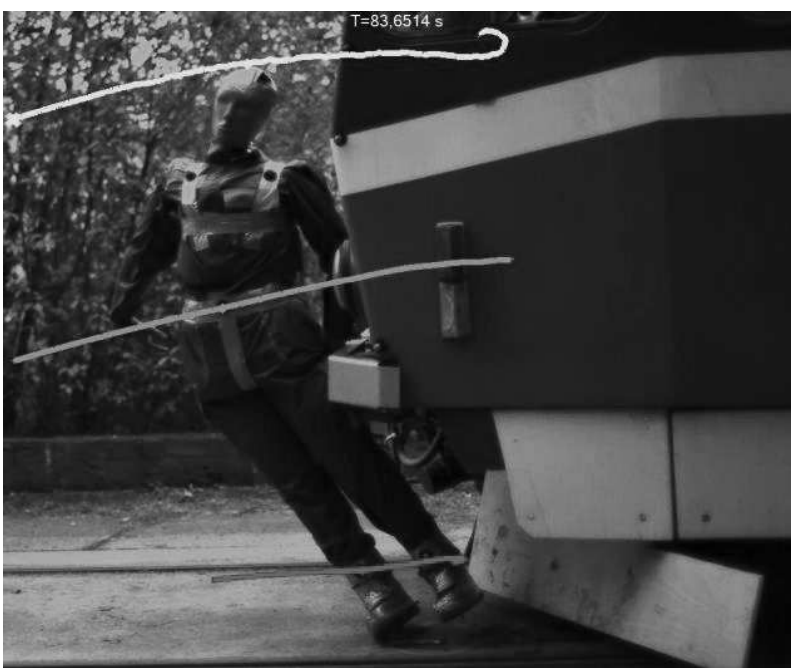

Fig. 4 Second stage-dummy's disentanglement from the tram's front (time 0.12s) 


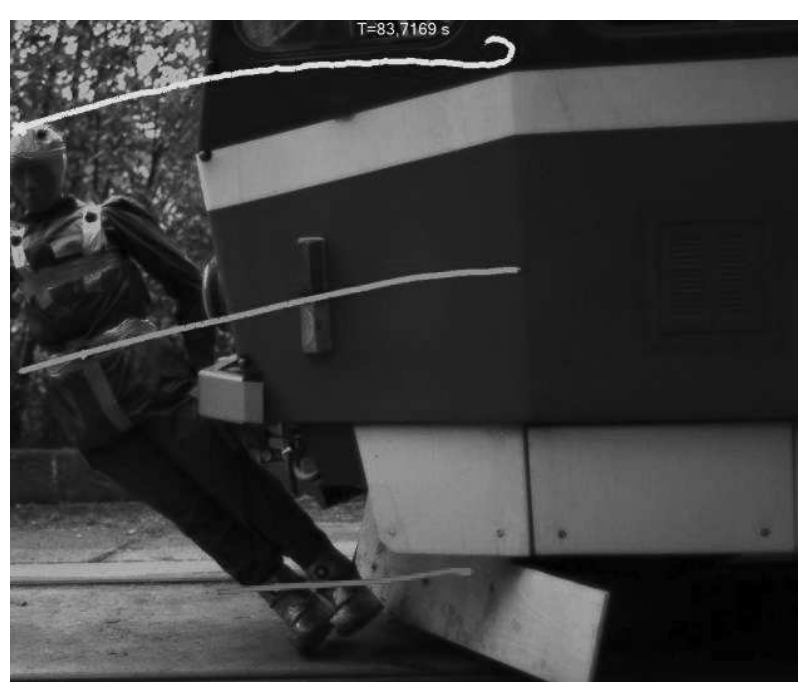

Fig. 5 Second stage-dummy's accelerated fall (time $0.19 s$ )

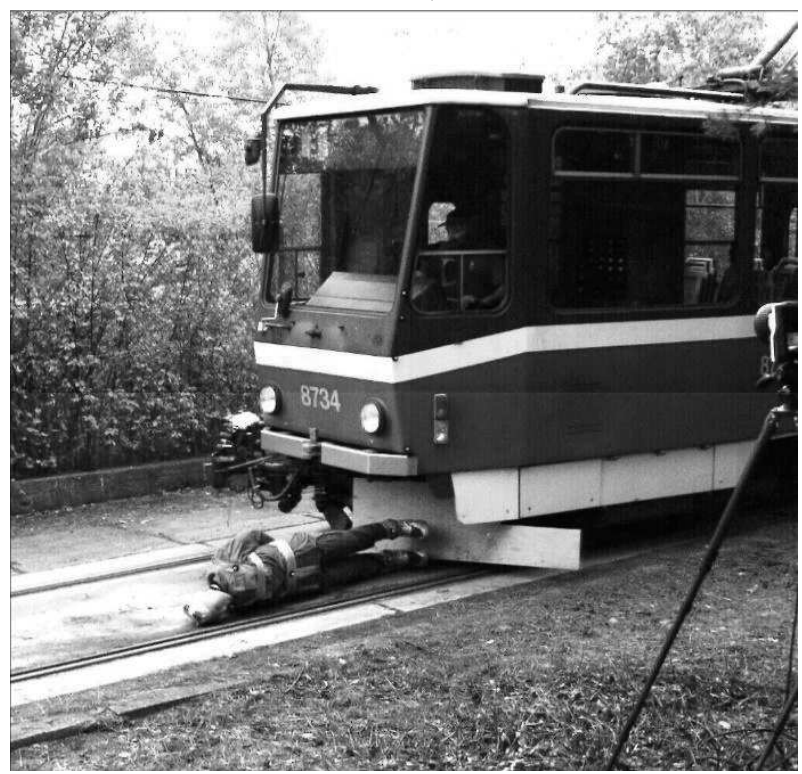

Fig. 6 Third stage-dummy's fall to the ground in front of the tram (time 0.48s)

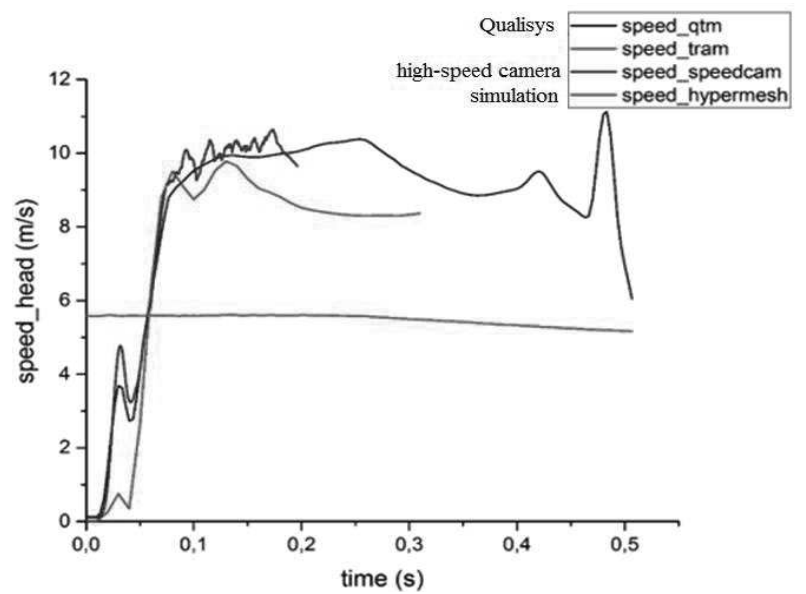

Fig. 7 Recorded and simulated curves of the dummy's head summation speed (summation speed = vector sum of components in directions of the used coordinates system)
An example of outputs from the measurements is provided on the chart below. It shows the speed of the head, a segment which is obviously extremely risky in the collisions of this type. The head speed curve established by the simulation is shown on the chart as well (Figure 7).

These curves justify the above-mentioned conclusion that the most devastating moment is when the dummy hits the ground. The curve shown in blue obtained from the analysis of a high-speed video recording is the most authentic as it is the recording with the highest sampling rate. On the black curve obtained from Qualisys, you can see the elimination of peaks which can be correctly estimated by directly comparing the recording with the highspeed recording. The clearly high degree of concordance of the first two stages of the collision event ascertained from the recorded and defined simulations is very interesting.

The head speed values at the times of the included figures can be seen in the chart,; at the time of $0.03 \mathrm{~s}$, when the primary impact of the head on the tram's front occurs, the head's summation of speed is about $5 \mathrm{~m} / \mathrm{s}$. As it moves in the opposite direction to the tram's driving direction (see the head marker trajectory in Figure 5), the impact speed in this case is the sum of the head speed and of the tram's driving speed, i.e. about $11 \mathrm{~m} / \mathrm{s}$. The head impact speed when the dummy hits the ground in front of the tram is definitely more than $12 \mathrm{~m} / \mathrm{s}$. Moreover, the head at this stage hits the rail corridor's very solid concrete structure, while during the collision with the tram's front the head hits a deformable sheet panel.

The tram's speed curve is also interesting. The speed started to slow down about 0.25 seconds after the primary impact.

On the black curve obtained from simulation of the performed test with a modified lower part of the tram's front (Figure 8), its deviation from the detected curve is visible from approximately $0.15 \mathrm{~s}$.

To evaluate the collision danger rates in terms of risk of injury, a series of respected criteria for different segments of the human body is used. In the case of the head, the most frequent criterion is $\mathrm{HIC}_{36}$ or, where applicable, $\mathrm{HIC}_{15}$ based on the nature of the collision. $\mathrm{HIC}_{36}$ is used in cases where there is no hard impact on the head. For the described case, the criterion $\mathrm{HIC}_{15}$ would be more appropriate, calculated using the following formula [2]:

$$
H I C=\left\{\left(t_{2}-t_{1}\right)\left[\frac{1}{t_{2}-t_{1}} \int_{t_{1}}^{t_{2}} a(t) d t\right]^{2,5}\right\}_{\max },
$$

where $a$ is the total acceleration actuating the head, $t_{l}$ is the initial time of collision and $t_{2}$ is the final time of the monitored part. Based on various resources, the crucial value of $\mathrm{HIC}_{15}$ fluctuates between 700 and 1000 [3]. The equipment used did not allow the actuating acceleration to be measured directly and the values of $\mathrm{HIC}_{15}$ which are presented below are therefore based on data acquired by numeric derivations of detected speed curves.

This fact illustrates the effect of the tram's front modification which results in impact on the calves and reduction in the dummy's load (Figure 9). 

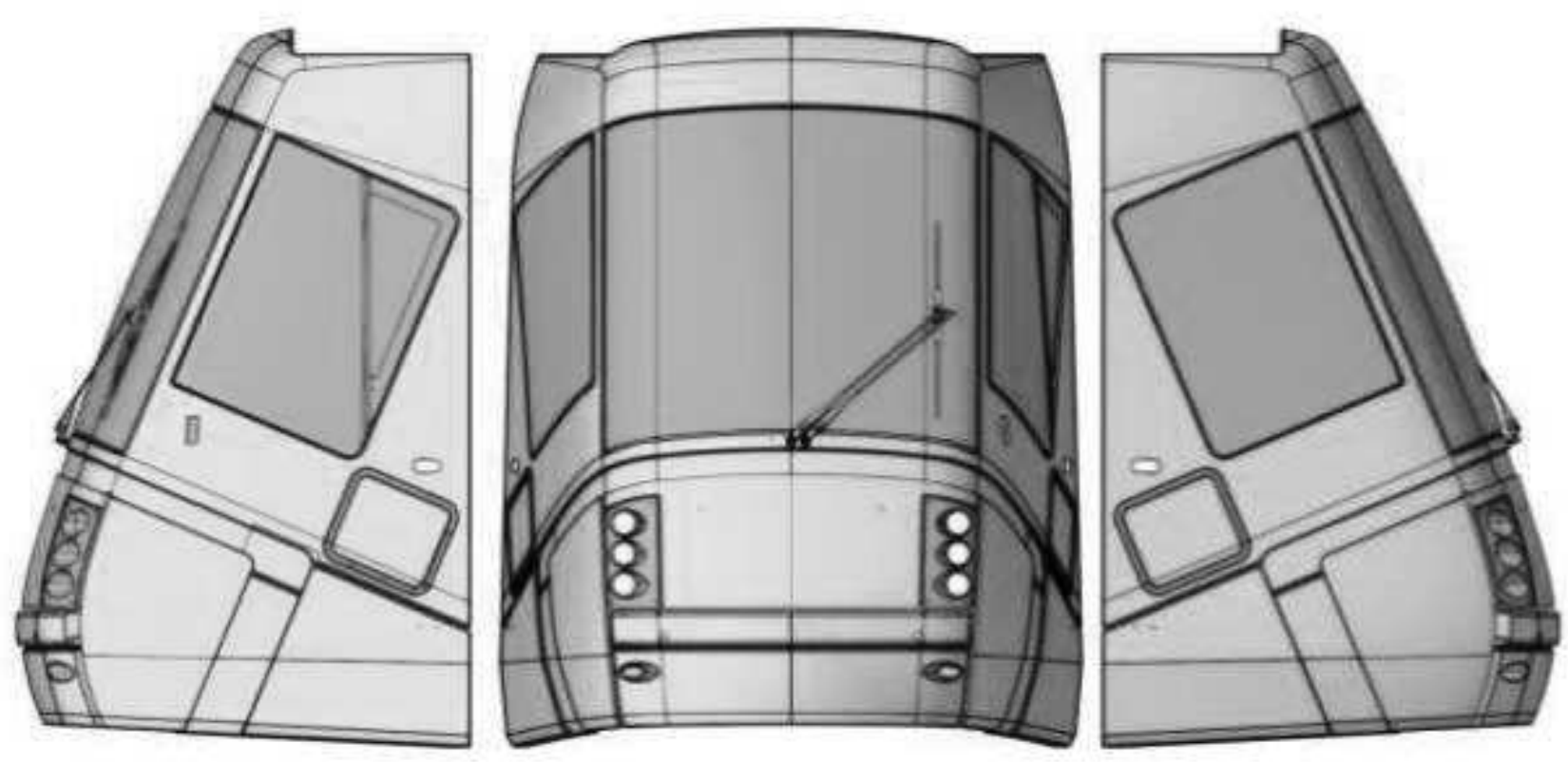

Fig. 8 Modified tram front

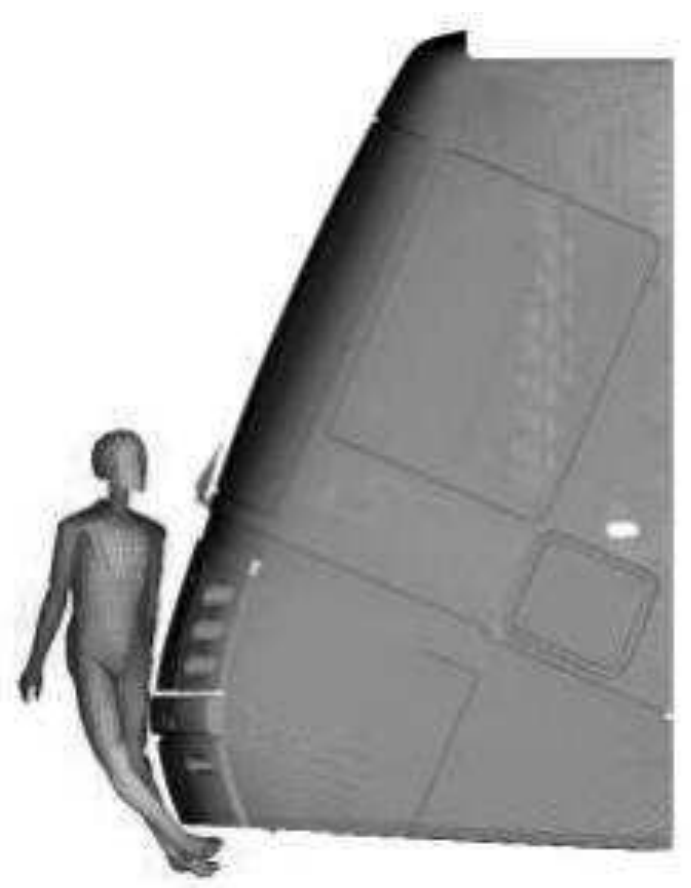

Fig. 9 Primary stage of impact of modified front on the dummy in the simulation

This way, the accelerated fall effect is removed and the dummy hits the ground under significantly less harmful conditions. For comparison, Table 1 shows $\mathrm{HIC}_{15}$ values at the time of primary impact at about 0.03 seconds, at the time when the dummy's head hits the ground (about $0.48 \mathrm{~s}$ ) and at the time of the dummy's head contact with the tram in the simulation, i.e. at the time of about $0.11 \mathrm{~s}$. At that time, the dummy was already moving with the tram, so the dummy way practically still towards the tram and the resulting impact speed was less than $4 \mathrm{~m} / \mathrm{s}$.
Tab. $1 \mathrm{HIC}_{15}$

\begin{tabular}{|c|c|c|}
\hline Time [s] & Collision stage & HIC $_{\mathbf{1 5}}$ \\
\hline 0.03 & $\begin{array}{c}\text { Dummy's head impact on } \\
\text { tram's front; experiment }\end{array}$ & $6,201.8$ \\
\hline 0.48 & $\begin{array}{c}\text { Dummy's head hitting the } \\
\text { ground; experiment }\end{array}$ & $24,008.3$ \\
\hline 0.11 & $\begin{array}{c}\text { Dummy's head impact on } \\
\text { tram's front; simulation }\end{array}$ & 741.6 \\
\hline
\end{tabular}

Although these values are only gross hypothetical values due to the lack of measured accelerations, it is quite clear that the way the tram's front "moulds" the impact victim has a crucial effect on the risk of the pedestrian being injured during a frontal collision.

\section{Results}

Partial results of the pilot study performed have been presented in the previous chapter in the interest of maintaining congruence of the text with the presented figures. The following text builds on the previous section and presents other findings in the form of annotated charts.

The ankle speed curves are shown in Figure 10. A direct comparison of the measured curves with the curves obtained in the simulation shows the effect of the modification of the tram's front. While the tested tram clearly pushes the dummy to the ground in the first stage of impact, thus bringing the dummy's movement in the tram's direction practically to a halt (time $0.05 \mathrm{~s}$ ), the front modification leads to a reduction in the dummy's load where the dummy is carried by the tram after the primary impact. Although this situation generates higher absolute and relative ankle speeds, it prevents the risk of the dummy getting under the tram during the next stages of the collision event. 


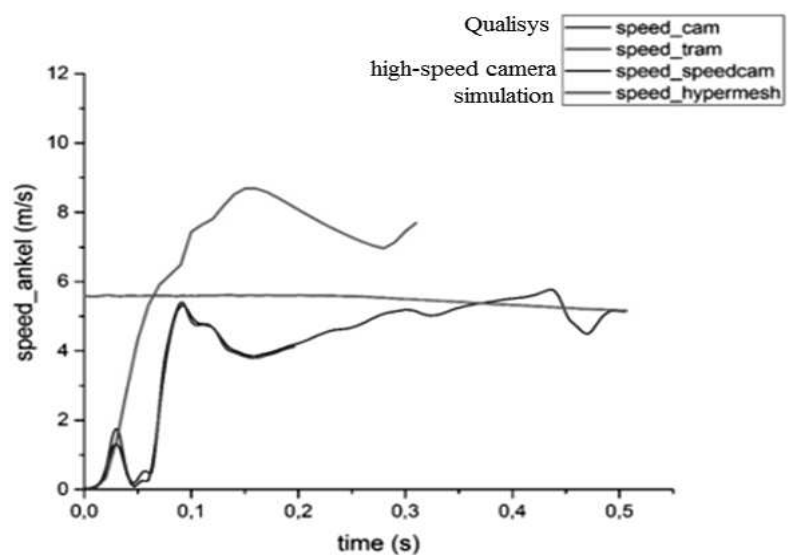

Fig. 10 Detected and simulated speed curves of the dummy's ankle

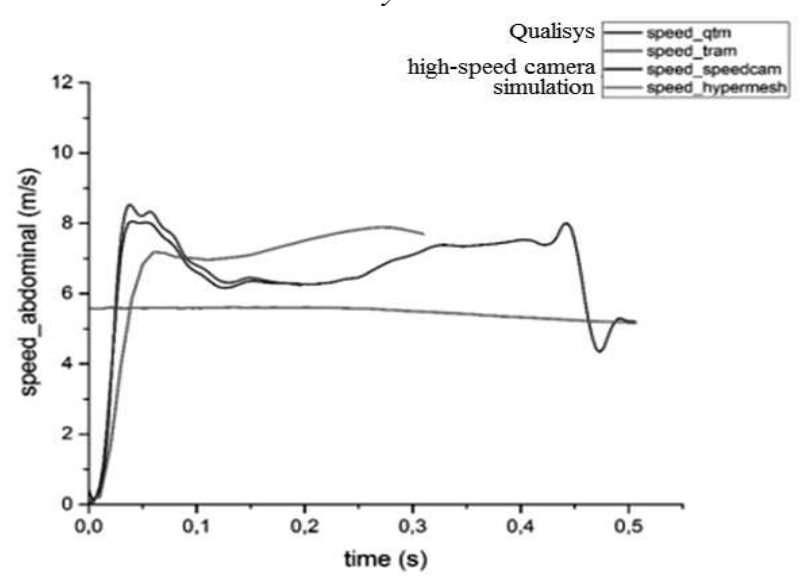

Fig. 11 Detected and simulated speed curves of the dummy's pelvis

The pelvis speed curves are shown in Figure 11. Thanks to the modified conditions of the primary impact, the simulation generated lower speeds for the dummy's pelvis, clearly in direct relation to the dummy being carried by the tram during the dummy's fall.

\section{Conclusion}

Although the article represents a pilot experiment supplemented by the first simulation of the monitored accident event, interesting results were obtained, hence the writing of this article. At this stage of our research, we are able to draw the following conclusions:

- When studying the collisions between pedestrians and trams, special attention must be paid to the entire course of the accident event, from the first contact to the pedestrian's impact on the ground.

- In all tested cases (three different speeds), three basic stages can be identified in the monitored course of collision event: primary impact stage, bounced fall stage and final impact stage.

- Friction between the soles and surface of the tram track plays an essential role in the development of the second and third stages.

- By introducing the situation where the dummy is carried by the tram immediately after the first stage of collision action, you can get significantly less harmful conditions in the dummy's impact on the ground at the final stage of accident.

- In all tested cases, the reaction time of the drivertram system was almost exactly $0.25 \mathrm{~s}$ (measured from the first contact of the tram front with the pedestrian).

- Although the Hybrid III dummy is not intended for studying direct sideways impacts, it provides highly reliable feedback which corresponds well with the virtual dummy used for studying collisions between pedestrians and passenger vehicles.

\section{Acknowledgements}

This article was written as part of the project CZ.02.1.01/0.0/0.0/16_026/0008401.

\section{References}

[1] Proceedings of the conference PASSIVE SAFETY of RAIL VEHICLES 2019. IFV Bahntechnik e.V. Berlin, 2019.

[2] Available at https://www.ifv-bahntechnik.de/literatur.pdf.

[3] RABLAU, G. (2016). Calculus in Crash Safety Tests: The Head Injury Criterion (HIC) Number. Kettering University, Michigan, 2016.

[4] Available at http://newb.kettering.edu/wp/experientialcalculus/wp-content/uploads/sites/15/2017/05/crash-safety-test.pdf.

[5] PURS, H. (2017). Mechanisms of Injuries in Extreme Load Areas. Charles University, Prague, 2017.

[6] Available at https://ftvs.cuni.cz/FTVS-2056version1-purs.pdf (auto report).

[7] KOVANDA K., HOLUB L., KOLAŘÍK L., KOLAŘÍKOVÁ M., VONDROUŠ M. (2012). Experimental Verification of FEM Simulation of GMAW Bead on Plate Welding., In: Manufacturing Technology, Volume 12, p. 30-33, Ústí nad Labem, 2012, ISSN 1213-2489.

[8] NOVÁK P., MEŠKO J., ŽMINDÁK M. (2013). Finite Element Implementation of Multi-Pass Fillet Weld with Phase Changes., In: Manufacturing Technology, Volume 13, p. 79-85, Ústí nad Labem, 2013, ISSN 1213-2489. 